УДК $517.55+517.96$

\title{
The Euler-Maclaurin Formula and Differential Operators of Infinite Order
}

\author{
Olga A. Shishkina* \\ Institute of Mathematics and Computer Science \\ Siberian Federal University \\ Svobodny, 79, Krasnoyarsk, 660041
}

Russia

Received 11.09.2014, received in revised form 02.10.2014, accepted 27.11.2014

We use methods of the theory of differential operators of infinite order for solving difference equations and for generalizing the Euler-Maclaurin formula in the case of multiple summation.

Keywords: indefinite summation, difference equations, differential operators of infinite order.

\section{Introduction}

The problem of summation of functions i.e the computation of the sum $S(x)=\sum_{t=0}^{x} \varphi(t)$ with a variable upper limit $x$ for a given function $\varphi(t)$ is a classical one. The sum of the sequence of powers of natural numbers $\varphi(t)=t^{2}$ was first computed by Jakob Bernoulli. His studies led to the development of several branches of the combinatorial analysis. Euler proposed a method, which reduces the problem to solving the difference equation

$$
f(x+1)-f(x)=\varphi(x),
$$

where $f(x)$ is an unknown function, and showed that $f(x)$ satisfies the differential equation

$$
D f(x)=\sum_{\mu=0}^{\infty} \frac{B_{\mu}}{\mu !} D^{\mu} \varphi(x),
$$

where $B_{\mu}$ are the Bernoulli numbers. If the functional series on the right-hand side of the equation can be integrated term by term, then from (2) we obtain the Euler-Maclaurin formula for the solution $f$ to equation (1), which expresses the unknown function in terms of the integral and derivatives of $\varphi(t)([1])$ :

$$
f(x)=\int \varphi(x) d x+\sum_{\mu=1}^{\infty} \frac{B_{\mu}}{\mu !} D^{\mu-1} \varphi(x) .
$$

Other approaches for solving the problem of summation we can find in [2]. Recently, there has been a surge of interest in problems of summation thanks to the development of symbolic algorithms of summation of rational functions in papes by S. A. Abramov [3] and S. P. Polyakov [4], who call these problems "the indefinite summation". In some cases, however, it is more appropriate to use a more general difference equation than (1).

*olga_a_sh@mail.ru

(C) Siberian Federal University. All rights reserved 
Denote $\delta$ a linear shift operator $\delta f(x)=f(x+1)$ and define a polynomial difference operator $P(\delta)=\sum_{\alpha=0}^{p} c_{\alpha} \delta^{\alpha}$, where $c_{\alpha}$ are constant coefficients. Consider the difference equation

$$
P(\delta) f(x)=\varphi(x)
$$

and show that some problems of indefinite summation can be reduced to solution of equations of this form. To solve equation (3) we use the methods of the theory of pseudodifferential operators namely the differential operators of infinite order [5].

With some additional conditions on the characteristic polynomial $P(z)=\sum_{\alpha=0}^{p} c_{\alpha} z^{\alpha}$ and the function $\varphi(x)$ in (3) we construct a differential operator of infinite order (see Theorem 1), and use it to obtain the following analogue of formula (2) for the solution $f(x)$ of the equation (3)

$$
D^{m} f(x)=\sum_{\mu=0}^{\infty} \frac{B_{\mu}(m)}{\mu !} D^{\mu} \varphi(x) .
$$

In this formula $B_{\mu}(m)$ are the generalized Bernoulli numbers, $m$ is the multiplicity of the root $z=1$ of the characteristic polynomial $P(z)$, and the functional series on the right-hand side converges for all $x$. If $P(\delta)=\delta-1$ and $m=1$ then the generalized Bernoulli numbers coincide with the Bernoulli numbers, and formula (4) with formula (2).

The indefinite summation problem in several variables can be stated differently. If $\varphi(t)$ is a polynomial in $t=\left(t_{1}, \ldots, t_{n}\right)$ and the summation is taken over the integer points of a rational convex polytope with variable faces, the multidimensional analogue of formula (4) is obtained in $[6,7]$.

In Section 2 of this paper we apply Theorem 1, or rather a corollary of it, to one problem of the multiple summation, namely the problem of finding the sum of the form $\sum_{\|t\| \leqslant x} \varphi(t)$, where $\varphi(t)=\varphi\left(t_{1}, \ldots, t_{n}\right)$ is a given function and $\|t\|=t_{1}+\ldots+t_{n}, t_{j}$ are non-negative integer numbers. Sometimes this summation is referred to as the "triangle" summation. If $\varphi$ is a function of one variable, then this sum can be written as

$$
S(x)=\sum_{\|t\| \leqslant x} \varphi(x-\|t\|) .
$$

We show that it satisfies (Proposition 2) the difference equation

$$
(\delta-1)^{n} S(x)=\varphi(x+n)
$$

that makes possible to apply formula (4) and solve the problem of indefinite summation (5). The analogue of the Euler-Maclaurin formula for the sum (5) is then (Theorem 2)

$$
S(x)=\sum_{\mu=0}^{n-1} \frac{B_{\mu}(m)}{\mu !} P_{n-\mu} \varphi(x+n)+\sum_{\mu=0}^{\infty} \frac{B_{\mu+n}(m)}{(\mu+n) !} D^{\mu} \varphi(x+n),
$$

where $P_{n-\mu} \varphi(x+n)$ is the antiderivative of order $n-\mu$ of the function $\varphi(x+n)$, and $B_{\mu}(m)$ are the generalized Bernoulli numbers. 


\section{Differential operators of infinite order and difference equations}

To derive formula (4) for the solution of the difference equation (3) and justify the convergence of the functional series in its right-hand side we need some information from the theory of pseudodiferential operators [5].

Let $\mathbb{C}^{d}$ be the complex $d$-dimensional space with points $z=\left(z_{1}, \ldots, z_{d}\right)$. Let $\operatorname{Exp}\left(\mathbb{C}^{d}\right)$ be the space of entire functions $\varphi(z)$ of exponential type i.e. entire functions satisfying inequality

$$
|\varphi(z)| \leqslant M e^{r|z|},
$$

where $M>0, r \geqslant 0$ are some numbers (its own for each function), and $|z|=\left|z_{1}\right|+\ldots+\left|z_{d}\right|$.

We denote $D=\left(D_{1}, \ldots D_{d}\right)$, where $D_{j}=\frac{\partial}{\partial z_{j}}$, and $D^{\alpha}=D_{1}^{\alpha_{1}} \ldots D_{d}^{\alpha_{d}}$ for the multi-index $\alpha=\left(\alpha_{1}, \ldots, \alpha_{d}\right)$.

Let $R>0$ be a fixed number and $r<R$ is arbitrary. We denote $\operatorname{Exp}_{R}\left(\mathbb{C}^{d}\right)$ the space of entire functions of exponential type $\varphi(z)$ such that for some $M>0$ and any $r<R$

$$
\left|D^{\alpha} \varphi(z)\right| \leqslant M r^{|\alpha|} e^{r|z|}
$$

for all multi-indexes $\alpha$ and for all $z \in \mathbb{C}^{d}$.

Let $A(\xi)$ be an analytic function in the polycylinder $U_{R}=\left\{\xi \in \mathbb{C}^{d}:\left|\xi_{j}\right|<R, j=1, \ldots, d\right\}$ with the expansion into the power series $A(\xi)=\sum_{|\alpha|=0}^{\infty} a_{\alpha} \xi^{\alpha}$. We can associate a differential operator of infinite order $A(D)$ with each analytic symbol $A(\xi)$ formally replacing the argument $\xi=\left(\xi_{1}, \ldots, \xi_{n}\right)$ by the differentiation symbol $D=\left(D_{1}, \ldots D_{d}\right)$. The function $A(\xi)$ is called the symbol of the operator $A(D)$.

The action of the operator $A(D)$ on the function $\varphi(z)$ is defined by

$$
A(D) \varphi(z)=\sum_{|\alpha|=0}^{\infty} a_{\alpha} D^{\alpha} \varphi(z)
$$

We denote $H\left(U_{R}\right)$ the space of holomorphic functions in $U_{R}$, and $A(\xi) \in H\left(U_{R}\right)$. It is well known (see [5], Theorem 5.1) that for any function $\varphi(z) \in \operatorname{Exp}_{R}\left(\mathbb{C}^{d}\right)$ the function $A(D) \varphi(z)$ is defined and belongs also to $\operatorname{Exp}_{R}\left(\mathbb{C}^{d}\right)$. Moreover, the mapping

$$
A(D): \operatorname{Exp}_{R}\left(\mathbb{C}^{d}\right) \rightarrow \operatorname{Exp}_{R}\left(\mathbb{C}^{d}\right)
$$

is continuous, and the set of the differential operators of infinite order $\{A(D)\}$ forms an algebra. ([5], Theorem 5.2). In particular, if in addition the (multiplicative) inverse function $A^{-1}(\xi)$ is analytic in $U_{R}$ then the operator $A^{-1}(D)$ is the inverse operator for $A(D)$.

First, we show that a polynomial difference operator $Q(\delta)=\sum_{|\alpha| \leqslant m} a_{\alpha} \delta^{\alpha}$, where $\delta^{\alpha}=$ $\delta_{1}^{\alpha_{1}} \ldots \delta_{d}^{\alpha_{d}}$, acts exactly as some differential operator of infinite order. Consider an entire function $Q\left(e^{\xi}\right)=Q\left(e^{\xi_{1}}, \ldots, e^{\xi_{d}}\right)$ in $\xi=\left(\xi_{1}, \ldots, \xi_{d}\right) \in \mathbb{C}^{d}$, then the differential operator of infinite order $Q\left(e^{D}\right)=Q\left(e^{D_{1}}, \ldots, e^{D_{d}}\right)$ is defined on the space of functions $\operatorname{Exp}\left(\mathbb{C}^{d}\right)$.

Proposition 1. $Q(\delta)=Q\left(e^{D}\right)$ on the space $\operatorname{Exp}\left(\mathbb{C}^{d}\right)$.

Proof. For any function $\varphi(z)$ of exponential type we have

$$
Q(\delta) \varphi(z)=\sum_{|\alpha| \leqslant m} a_{\alpha} \delta^{\alpha} \varphi(z)=\sum_{|\alpha| \leqslant m} a_{\alpha} \varphi(z+\alpha),
$$


and expanding $\varphi(z+\alpha)$ in powers of $\alpha$, we obtain

$$
Q(\delta) \varphi(z)=\sum_{|\alpha| \leqslant m} a_{\alpha} \sum_{|k|=0}^{\infty} \frac{\varphi^{(k)}(z)}{k !} \alpha^{k}=\sum_{|\alpha| \leqslant m} a_{\alpha} \sum_{|k|=0}^{\infty} \frac{(\alpha D)^{k}}{k !} \varphi(z) .
$$

On the other hand, $Q\left(e^{\xi}\right)=\sum_{|\alpha| \leqslant m} a_{\alpha}\left(e^{\xi}\right)^{\alpha}=\sum_{|\alpha| \leqslant m} a_{\alpha} e^{\alpha \xi}=\sum_{|\alpha| \leqslant m} a_{\alpha} \sum_{|k|=0}^{\infty} \frac{(\alpha \xi)^{k}}{k !}$. Therefore, $Q\left(e^{D}\right) \varphi(z)=\sum_{|\alpha| \leqslant m} a_{\alpha} \sum_{|k|=0}^{\infty} \frac{(\alpha D)^{k}}{k !} \varphi(z)$.

Thus, $Q(\delta) \varphi(z)=Q\left(e^{D}\right) \varphi(z)$ for any $\varphi(z) \in \operatorname{Exp}\left(\mathbb{C}^{d}\right)$.

We now define the needed differential operators of infinite order. Denote by $\aleph$ the set of all differential operators of infinite order with analytic symbol in the circle $U_{R}$. They form an algebra isomorphic to the algebra of all analytic in $U_{R}$ functions. Let $z=1$ be a root of multiplicity $m$ of the characteristic polynomial $P(z)$ of the difference equation (4), the multiplicity $m$ can be any integer from 0 to $p$. We construct a differential operator of infinite order associated with this polynomial. To this end, denote by $R$ the distance from $\xi=0$ to the nearest zero of the function $P\left(e^{\xi}\right)$ and consider the function $A(\xi)=\frac{\xi^{m}}{P\left(e^{\xi}\right)}=\sum_{\mu=0}^{\infty} \frac{B_{\mu}(m)}{\mu !} \xi^{\mu}$, where $m$ is the multiplicity of the root $\xi=0$. We shall call the numbers $B_{\mu}(m)$ the generalized Bernoulli numbers. The function $A(\xi)$ is holomorphic in the disk $U_{R}=\left\{\xi \in \mathbb{C}^{1}:|\xi|<R\right\}$, therefore the differential operator $A(D)$ is defined on the space $\operatorname{Exp}_{R}(\mathbb{C})$.

It is obvious that in the algebra $\aleph$ we have

$$
P\left(e^{D}\right) A(D)=D^{m}
$$

Indeed, the operators $P\left(e^{D}\right)$ and $A(D)$ belong to the algebra $\aleph$, and since for their symbols $P\left(e^{\xi}\right)$ and $A(\xi)$ in the disk $U_{R}$ the equality $P\left(e^{\xi}\right) A(\xi)=\xi^{m}$ is true, for any $\varphi(z) \in \operatorname{Exp}_{R}(\mathbb{C})$ we have $P\left(e^{D}\right) A(D) \varphi(z)=D^{m} \varphi(z)$.

Theorem 1. Let $\varphi(z) \in \operatorname{Exp}_{R}(\mathbb{C})$, and $z=1$ be a root of multiplicity $m$ of the characteristic polynomial. The function $f(z) \in \operatorname{Exp}_{R}(\mathbb{C})$ is a solution to the difference equation (3) if and only if $f(z)$ is a solution to the differential equation

$$
D^{m} f(z)=A(D) \varphi(z)
$$

where $A(D)=\sum_{\mu=0}^{\infty} \frac{B_{\mu}(m)}{\mu !} D^{\mu}$, and $B_{\mu}(m)$ are the generalized Bernoulli numbers.

Proof. First, we prove the necessity. Let $\varphi(z) \in \operatorname{Exp}_{R}(\mathbb{C})$ and $f(z) \in \operatorname{Exp}_{R}(\mathbb{C})$ is the solution to the equation (3). By Proposition 1 we have $P(\delta)=P\left(e^{D}\right)$. From (3) we obtain $P\left(e^{D}\right) f(z)=\varphi(z)$. Then, after multiplication by $A(D)$ we have $P\left(e^{D}\right) A(D) f(z)=$ $A(D) \varphi(z)$. According to $(7)$ we get $D^{m} f(z)=A(D) \varphi(z)$, i.e. $f$ satisfies $(8)$.

To prove the sufficiency, we prove that if the function $u(z) \in \operatorname{Exp}_{R}(\mathbb{C})$ then any its antiderivative belongs to $\operatorname{Exp}_{R}(\mathbb{C})$. In fact, if we suppose that $u(z) \in \operatorname{Exp}_{R}(\mathbb{C})$ and its antiderivative $\widetilde{u}(z) \notin \operatorname{Exp}_{R}(\mathbb{C})$, then for any $M>0$ and $r<R$ there are $\alpha_{0}$ and a point $z_{0} \in \mathbb{C}$ such that $\left|D^{\alpha_{0}} \widetilde{u}\left(z_{0}\right)\right|>M r^{\left|\alpha_{0}\right|} e^{r\left|z_{0}\right|}$, then $\left|D^{\alpha_{0}-1} u\left(z_{0}\right)\right|>M r^{\left|\alpha_{0}\right|} e^{r\left|z_{0}\right|}$ and $\left|D^{\alpha_{0}-1} u\left(z_{0}\right)\right|>M r r^{\left|\alpha_{0}-1\right|} e^{r\left|z_{0}\right|}$. This means that $u(z)$ does not belong to $\operatorname{Exp}_{R}(\mathbb{C})$.

Now we prove that if $f(z)$ is a solution to the differential equation (8) then $f(z)$ is a solution 
to the difference equation (3). Since $D^{m} f(z)=A(D) \varphi(z)$, and according to the equality (7), we have $D^{m}=P\left(e^{D}\right) A(D), P\left(e^{D}\right) A(D) f(z)=A(D) \varphi(z)$. Apply the operator $A^{-1}(D)$ to the both sides of the last equality to obtain $P\left(e^{D}\right) f(z)=\varphi(z)$, and taking into account Proposition 1 we get

$$
P(\delta) f(z)=\varphi(z) .
$$

Therefore, if $\varphi(z) \in \operatorname{Exp}_{R}(\mathbb{C})$ then the solution $f(z)$ to the difference equation (3) is also in this space.

Successively integrating (8), we obtain as a corollary the formula for the solution to the difference equation (3):

Corollary of Theorem 1 . Under the conditions of Theorem 1 the function $f(z) \in \operatorname{Exp}_{R}(\mathbb{C})$ is a solution to (3) if and only if

$$
f(z)=\sum_{\mu=0}^{m-1} \frac{B_{\mu}(m)}{\mu !} P_{m-\mu} \varphi(z)+\sum_{\mu=0}^{\infty} \frac{B_{\mu+m}(m)}{(\mu+m) !} D^{\mu} \varphi(z),
$$

where $P_{n-\mu} \varphi(z)$ is an antiderivative of order $n-\mu$ of the function $\varphi(z)$, and $B_{\mu}(m)$ are the generalized Bernoulli numbers.

Example 1. As en example of applaying of the Theorem 1 we solve the equation (3) for $\varphi(z)=$ $q^{z}, q>0$. Since $q^{z}=e^{z \ln q}, \varphi(z) \in \operatorname{Exp}_{R}(\mathbb{C})$ for $q<e^{R}$. Let $m$ be the multiplicity of the root $z=1$ of the characteristic polynomial $P(z)$, then by Theorem $1 D^{m} f(z)=\sum_{\mu=0}^{\infty} \frac{B_{\mu}(m)}{\mu !} D^{\mu} q^{z}$. Rewrite this equality

$$
D^{m} f(z)=\sum_{\mu=0}^{\infty} \frac{B_{\mu}(m)}{\mu !}(\ln q)^{\mu} q^{z}=q^{z} \sum_{\mu=0}^{\infty} \frac{B_{\mu}(m)}{\mu !}(\ln q)^{\mu}=\frac{q^{z}(\ln q)^{m}}{P\left(e^{\ln q}\right)}=\frac{q^{z} \ln ^{m} q}{P(q)} .
$$

Integrating the last equality, we obtain a formula for the solution of the equation $P(\delta) f(z)=q^{z}$ for $m \geqslant 1$

$$
f(z)=\frac{q^{z}}{P(q)}+C_{1} z^{m-1}+\ldots+C_{m-1} z+C_{m}
$$

where $C_{j}$ are arbitrary constants.

\section{A generalization of the Euler-Maclaurin formula}

In this section we apply Theorem 1 to the problem of finding the sum (5). Similar sums appear, for example, in some problems of the enumerative combinatorial analysis and are called the sum with linear constraints on the summation indexes $[8,9]$.

If $\varphi(x)$ is a function of one variable, then the sum (5) can be written as

$$
S(x)=\sum_{k=0}^{x} C_{k+n-1}^{k} \varphi(x-k),
$$

where $C_{k+n-1}^{k}=\frac{(k+n-1) !}{k !(n-1) !}$ is the number of non-negative integer solutions $t=\left(t_{1}, \ldots, t_{n}\right)$ to $t_{1}+\ldots+t_{n}=k$. 
The right-hand side of (9) involves a single sum, but in contrast to the classical case, we need to sum over $k$ the function $C_{k+n-1}^{k} \varphi(x-k)$, which depends on the parameter $x$. This is similar to the problem of calculating an integral with a parameter and a variable upper limit. For example, for $n=2$ this sum can be written as

$$
S(x)=\sum_{k=0}^{x}(k+1) \varphi(x-k)=\sum_{t=0}^{x}(x-t+1) \varphi(t)
$$

Theorem 2. If $\varphi(z) \in \operatorname{Exp}_{2 \pi}(\mathbb{C})$ then for the sum (9) we have

$$
S(x)=\sum_{\mu=0}^{n-1} \frac{B_{\mu}(n)}{\mu !} P_{n-\mu} \varphi(x+n)+\sum_{\mu=0}^{\infty} \frac{B_{\mu+n}(n)}{(\mu+n) !} D^{\mu} \varphi(x+n),
$$

where $B_{\mu}(n)=\sum_{\|k\|=\mu} \frac{\|k\| !}{k !} B_{k}, \quad k=\left(k_{1}, \ldots, k_{n}\right), \quad k !=k_{1} ! \ldots k_{n} !, \quad B_{k}=B_{k_{1}} \cdot \ldots \cdot B_{k_{n}}, \quad$ and $B_{k_{j}}$ are the Bernoulli numbers, $P_{n-\mu}$ is the antiderivative of order $n-\mu$.

For the proof we need the following proposition.

Proposition 2. The function $S(x)$ defined by (9) satisfies the difference equation

$$
(\delta-1)^{n} S(x)=\varphi(x+n) .
$$

Proof. For $t=1,2, \ldots, n-1$ we have the formula

$$
\begin{aligned}
& (\delta-1)^{t} S(x)=\sum_{k=0}^{x+t} C_{n+k-t-1}^{k} \varphi(x+t-k) . \text { Let } t=n-1, \text { then } \\
& (\delta-1)^{n-1} S(x)=\sum_{k=0}^{x+n-1} C_{k}^{k} \varphi(x+n-1-k)=\varphi(0)+\varphi(1)+\ldots+\varphi(x+n-1) .
\end{aligned}
$$

Applying the operator $\delta$ to (12) we obtain

$$
\delta(\delta-1)^{n-1} S(x)=\sum_{k=0}^{x+n} C_{k}^{k} \varphi(x+n-k)=\varphi(0)+\varphi(1)+\ldots+\varphi(x+n-1)+\varphi(x+n) .
$$

If we subtract equation (12) from (13), we obtain

$$
(\delta-1)^{n} S(x)=\delta(\delta-1)^{n-1} S(x)-(\delta-1)^{n-1} S(x)=\varphi(x+n)
$$

Proof of Theorem 2. According to Proposition 2 the sum (9) satisfies the difference equation (11) with the characteristic polynomial $P(z)=(z-1)^{n}$. For the function $P\left(e^{\xi}\right)=\left(e^{\xi}-1\right)^{n}$ the point $\xi=0$ is a root of order $n$, and the distance from it to the nearest root is $R=2 \pi$. It is not difficult to verify that the function $A(\xi)=\frac{\xi^{n}}{\left(e^{\xi}-1\right)^{n}}$ is analytic at the point $\xi=0$, and the series $A(\xi)=\sum_{\mu=0}^{\infty} \frac{B_{\mu}(n)}{\mu !} \xi^{\mu}$ converges in the circle $U_{2 \pi}=\{\xi \in \mathbb{C}:|\xi|<2 \pi\}$.

The differential operator of infinite order corresponding to the function $A(\xi)$ is defined on 
functions from the space $\operatorname{Exp}_{2 \pi}(\mathbb{C})$, that is why by Theorem 1 we see that for $\varphi(z) \in \operatorname{Exp}_{2 \pi}(\mathbb{C})$ any solution $S(z)$ to the equation $(9)$ in $\operatorname{Exp}_{2 \pi}(\mathbb{C})$ satisfies the equation

$$
D^{n} S(z)=\sum_{\mu=0}^{\infty} \frac{B_{\mu}(n)}{\mu !} D^{\mu} \varphi(z+n) .
$$

If we denote by $P_{1} u(z)=\int_{z_{0}}^{z} u(\tau) d \tau$ the antiderivative of the function $u(z)$, then we obtain the following formula on the real axis for the required sum

$$
S(x)=P_{n} \sum_{\mu=0}^{\infty} \frac{B_{\mu}(n)}{\mu !} D^{\mu} \varphi(x+n)=\sum_{\mu=0}^{n-1} \frac{B_{\mu}(n)}{\mu !} P_{n-\mu} \varphi(x+n)+\sum_{\mu=0}^{\infty} \frac{B_{\mu+n}(n)}{(\mu+n) !} D^{\mu} \varphi(x+n),
$$

where $P_{n-\mu} \varphi(x+n)$ is an antiderivative of order $n-\mu$ of the function $\varphi(x+n)$.

The generalized Bernoulli numbers $B_{\mu}(n)$ can be found by means of classical ones $B_{\mu}$. It is known that the Bernoulli numbers appear as the coefficients in the power series expansion of the function $\frac{\xi}{\left(e^{\xi}-1\right)}=\sum_{\mu=0}^{\infty} \frac{B_{\mu}}{\mu !} \xi^{\mu}$. Raising this equality into power $n$, we get $B_{\mu}(n)=\sum_{\|k\|=\mu} \frac{\|k\| !}{k !} B_{k}$, where $k=\left(k_{1}, \ldots, k_{n}\right), k !=k_{1} ! \ldots k_{n} !, B_{k}=B_{k_{1}} \cdot \ldots \cdot B_{k_{n}}$. Note that for $n=1$ formula (14) is the Euler-Maclaurin formula.

Example 2. For the sum of the geometric series $S(x)=\sum_{k=0}^{x} q^{k}$, by Proposition 2, this problem is reduced to the solution of the equation $(\delta-1) S(x)=q^{x+1}, S(0)=1$. In this case $P(z)=z-1$, $R=2 \pi$, and $q<e^{2 \pi}$. According to Example 1, we have $S(x)=\frac{q^{x+1}}{q-1}+C$. Using $S(0)=1$, we find the constant $C$, and see that the required sum is equal to $S(x)=\frac{q^{x+1}-1}{q-1}$.

Example 3. The problem of finding the sum (9) for $\varphi(x)=q^{x}$ with $n=2$, i.e. $S(x)=$ $\sum_{k=0}^{x}(k+1) q^{x-k}$ is less trivial. By Proposition 2 we have to solve the equation $(\delta-1)^{2} S(x)=$ $q^{x+2}$ with the initial conditions $S(0)=1, \quad S(1)=q+2$. In this case $P(z)=(z-1)^{2}, R=2 \pi$, and $q<e^{2 \pi}$. According to Example 1 we obtain $S(x)=\frac{q^{x+2}}{(q-1)^{2}}+C_{1} z+C_{2}$. The constants can be found by using the initial data. Thus,

$$
S(x)=\frac{q^{x+2}}{(q-1)^{2}}-\frac{1}{q-1} x+\frac{1-2 q}{(q-1)^{2}} .
$$

\section{References}

[1] A.O.Gelfond, Calculus of finite differences, Moscow, Nauka, 1977 (in Russian).

[2] G.Hardy, Divergent series, Oxford University Press, London, 1949.

[3] S.A.Abramov, On the summation of rational functions, USSR Comput. Math. Math. Phys., 11(1971), no. 4, 324-330.

[4] S.P.Polyakov, Indefinite summation of rational functions with factorization of denominators, Programming and Computer Software, 37(2011), no. 6, 322-325. 
[5] Yu.A.Dubinskyi, The Cauchy problem in the complex domain, Moscow, Izdatelstvo MEI, 1996 (in Russian).

[6] M.Brion, M.Vergne, Lattice points in simple polytopes, J. American Math. Soc., 10(1997), no. $2,371-392$.

[7] M.Brion, M.Vergne, Residue formulae, vector partition functions and lattice points in rational polytopes, J. American Math. Soc., 10(1997), no. 4, 797-833.

[8] G.P.Egorychev, Integral Representation and the Computation of Combinatorial Sums, AMS, Providence, RI, 1984.

[9] E.K.Leynartas, Multidimensional Hadamard composition and sums with linear constraints on the summation indices, Sib. Math. J., 30(1989), no. 2, 250-255.

\section{Формула Эйлера-Маклорена и дифференциальные операторы бесконечного порядка}

\section{Ольга А. Шишкина}

Методы теории дифференциальных операторов бесконечного порядка использованы для нахождения решений разностных уравнений и обобщения формулы Эйлера-Маклорена на случай кратного суммирования.

Ключевые слова: неопределенное суммирование, разностные уравнения, псевдодифференциалъные операторы. 